\title{
Evaluation of the protective effects of curcuminoid (curcumin and bisdemethoxycurcumin)-loaded liposomes against bone turnover in a cell-based model of osteoarthritis
}

\author{
This article was published in the following Dove Press journal: \\ Drug Design, Development and Therapy \\ 20 April 2015 \\ Number of times this article has been viewed
}

\author{
Chih-Chang Yeh ${ }^{1,2}$ \\ Yu-Han $\mathrm{Su}^{3}$ \\ Yu-Jhe Lin $^{3}$ \\ Pin-Jyun Chen ${ }^{3}$ \\ Chung-Sheng Shi' \\ Cheng-Nan Chen ${ }^{3, *}$ \\ Hsin-I Chang,* \\ 'Graduate Institute of Clinical Medical \\ Sciences, College of Medicine, Chang \\ Gung University, Taoyuan 333, Taiwan, \\ Republic of China; ${ }^{2}$ Orthopaedic \\ Department, Chiayi Branch, \\ Taichung Veterans General Hospital, \\ Chiayi, Taiwan, Republic of China; \\ ${ }^{3}$ Department of Biochemical Science \\ and Technology, National Chiayi \\ University, Chiayi, Taiwan, Republic \\ of China \\ *These authors contributed equally \\ to this work
}

\begin{abstract}
Curcumin (Cur) and bisdemethoxycurcumin (BDMC), extracted from Curcuma longa, are poorly water-soluble polyphenol compounds that have shown anti-inflammatory potential for the treatment of osteoarthritis. To increase cellular uptake of Cur and BDMC in bone tissue, soybean phosphatidylcholines were used for liposome formulation. In this study, curcuminoid (Cur and BDMC)-loaded liposomes were characterized in terms of particle size, encapsulation efficiency, liposome stability, and cellular uptake. The results show that there is about $70 \%$ entrapment efficiency of Cur and BDMC in liposomes and that particle sizes are stable after liposome formation. Both types of liposome can inhibit macrophage inflammation and osteoclast differential activities. In comparison with free drugs (Cur and BDMC), curcuminoid-loaded liposomes were less cytotoxic and expressed high cellular uptake of the drugs. Of note is that Cur-loaded liposomes can prevent liposome-dependent inhibition of osteoblast differentiation and mineralization, but BDMC-loaded liposomes could not. With interleukin (IL)-1 $\beta$ stimulation, curcuminoid-loaded liposomes can successfully downregulate the expression of inflammatory markers on osteoblasts, and show a high osteoprotegerin (OPG)/ receptor activator of nuclear factor $\kappa \mathrm{B}$ ligand (RANKL) ratio to prevent osteoclastogenesis. In the present study, we demonstrated that Cur and BDMC can be successfully encapsulated in liposomes and can reduce osteoclast activity and maintain osteoblast functions. Therefore, curcuminoid-loaded liposomes may slow osteoarthritis progression.
\end{abstract}

Keywords: liposome, osteoarthritis, curcuminoid, OPG/RANKL ratio, bone turnover

\section{Introduction}

Bone turnover is a continuous process of bone resorption and formation for the purpose of maintaining normal bone mass. Bone turnover involves the old bone resorption by osteoclasts and new bone formation by osteoblasts. ${ }^{1-3}$ Osteoclasts, bone-resorbing multinucleated cells, are generated from hematopoietic monocyte/macrophage precursor cells through differentiation processes. ${ }^{4}$ Differentiated and matured osteoclasts are characterized by high expression of tartrate-resistant acid phosphatase (TRAP) and cathepsin K. TRAP is a metallophosphoesterase participating in osteoclast-mediated bone turnover. ${ }^{5}$ Cathepsin $\mathrm{K}$ is an essential cysteine proteinase in collagenous bone matrix degradation. Cathepsin $\mathrm{K}$ can efficiently activate TRAP through proteolytic processing and these two proteins colocalize in osteoclasts to increase bone resorptive activity. ${ }^{6}$ Bone formation involves the proliferation, differentiation, and mineralization of osteoblasts. Active osteoblasts produce bone formation markers which are expressed
Correspondence: Cheng-Nan Chen; Hsin-I Chang

Department of Biochemical Science and Technology, National Chiayi University, 300 University Road, Chiayi, Taiwan, Republic of China

Email cnchen@mail.ncyu.edu.tw; hchang@mail.ncyu.edu.tw 
during different phases of osteoblast development. ${ }^{7}$ Bone formation markers include collagen I, alkaline phosphatase (ALP), osteocalcin, osteopontin, and other molecules. ${ }^{8-10}$ During the bone loss process, high concentrations of matrix metalloproteinases (MMPs) (involved in the initial destruction of collagen) and a high ratio of receptor activator of nuclear factor $\kappa \mathrm{B}$ ligand (RANKL)/osteoprotegerin (OPG) are observed. ${ }^{11,12}$ RANKL, a member of the tumor necrosis factor (TNF) family, can activate nuclear factor- $\kappa \beta$ ligand (NF- $\mathrm{KB}$ ) through RANK-RANKL specific binding to stimulate the survival, differentiation, and activation of osteoclasts. ${ }^{13}$ OPG, a membrane-bound TNF-related factor, works as a decoy receptor for RANKL and therefore the binding of OPG to RANKL prevents RANK activation and subsequent osteoclastogenesis. ${ }^{14,15}$ The RANKL/OPG ratio is an index of osteoclastogenic stimulus. ${ }^{16}$

Osteoarthritis (OA) is a progressive bone disease characterized by degeneration of articular cartilage, synovial inflammation, and changes in periarticular and subchondral bone. Recently, OA has been often discussed when talking about bone turnover. ${ }^{17}$ In the early stages of OA, bone turnover rate and subchondral bone loss are elevated. OA that involves a number of inflammatory cytokines, chemokines, and other mediators such as interleukin (IL)-1 $\beta$, IL-6, IL-11, IL-17 and TNF- $\alpha$, can induce bone loss by increasing RANKL expression and decreasing OPG production on osteoblasts and stromal cells. ${ }^{18,19}$ A 50\% decrease in cartilage pathology score, based on the gold-standard Mankin score, was reported on extensive inhibition of bone resorption, implicating the importance of osteoclast function and bone turnover in the pathogenesis of OA. ${ }^{20}$ Therefore, bone turnover is likely to play a pivotal role in the pathogenesis of OA.

Curcuminoids are yellow, lipid-soluble polyphenols extracted from the rhizome of turmeric (Curcuma longa). Curcuminoids consist of three principal active components: curcumin (Cur), demethoxycurcumin (DMC), and bisdemethoxycurcumin (BDMC). ${ }^{21}$ Curcuminoids have been shown to suppress oxidative stress and inflammatory markers in diabetic patients and induce apoptotic cell death of a wide variety of tumor cells. ${ }^{22-26}$ In 2009, Funk et al and Bharti et al reported that Cur is an attractive agent to chronic inflammatory diseases like neurodegenerative diseases, cardiovascular diseases, rheumatoid arthritis, and other arthritic diseases. ${ }^{27,28}$ Although some studies have reported that Cur inhibits RANKL activation in osteoclast precursors and suppresses osteoclastogenesis, ${ }^{29,30}$ it is interesting to note that the functions and mechanisms of curcuminoid in regulating bone turnover have not fully been investigated. ${ }^{31}$ In addition, light sensitivity, low water solubility, and poor oral bioavailability restrict the therapeutic value of curcuminoids. Based on recorded data, Cur and DMC are less stable than BDMC. ${ }^{32}$ To overcome the problems related to curcuminoid solubility, stability, and bioavailability, many nanocarriers have been attempted, including liposomes, polymeric nanoparticles, biodegradable microspheres, cyclodextrin, and hydrogels. ${ }^{33-35}$ Liposomes are artificially prepared vesicles made of phospholipid bilayers. ${ }^{35}$ Several techniques, such as the Bangham method, the detergent depletion method, the ether/ethanol injection method, reverse-phase evaporation, and the emulsion method, have been reported for preparing liposomes with high entrapment efficiency, narrow particle size distribution, and long-term stability. ${ }^{35,36}$ Previous studies have demonstrated that Cur encapsulation in liposomes can improve the drug bioactivities. ${ }^{37,38}$ In this study, we used liposomes encapsulating Cur and BDMC individually with nanoparticle sizes to improve the drug solubility and bioavailability and then investigate their therapeutic approach in an in vitro model of OA.

\section{Materials and methods Cell culture and reagents}

Cur was purchased from Alfa Aesar (Heysham, England). BDMC was obtained from TCI, Japan. Soybean phosphatidylcholine (SPC) (Phospholipon 90G) were purchased from American Lecithin Company (Germany). Alkaline Phosphatase Colorimetric Assay kit, Trichrome Stain (Masson) kit, Acid Phosphatase Leukocyte Kit, and all chemicals of reagent grade were purchased from Sigma-Aldrich Co. (St Louis, MO, USA). All cell culture materials were purchased from Thermo Fisher Scientific (Waltham, MA, USA) and all solvents used were of analytical grade (J.T. Baker, USA). Cytokines were from Prospec. Mouse osteoblast-like cells (7F2) and macrophages (RAW264.7) were obtained from the Food Industry Research and Development Institute (Taiwan). Cell lines 7F2 and RAW264.7 were cultured in Dulbecco's Modified Eagle's Medium (DMEM) supplemented with $10 \% \mathrm{v} / \mathrm{v}$ fetal bovine serum (FBS), 100 units/mL penicillin, and $100 \mu \mathrm{g} / \mathrm{mL}$ of streptomycin. Cells were maintained at $37^{\circ} \mathrm{C}$ with $5 \% \mathrm{CO}_{2}$ in a humidified incubator.

\section{Liposome}

Liposomes loading with Cur or BDMC were prepared using the modified thin-film hydration method. ${ }^{39}$ Phospholipids were dissolved in chloroform and mixed with a range of molar ratios of cholesterol to lipid (30:70, 1:2, 40:60, 45:55). 
Cur or BDMC (2 mM) dissolved in ethanol was added into the phospholipid solution in a round-bottom flask. The solvent was evaporated with a rotary evaporator at $45^{\circ} \mathrm{C}$ and vacuum dried to form a dry lipid film. The lipid film was then hydrated with distilled water. The hydrated solution was downsized by sequence passing through $400 \mathrm{~nm}, 200 \mathrm{~nm}$, and $100 \mathrm{~nm}$ polycarbonate membranes using an extruder (Avanti Mini Extruder; Alabama, USA) to obtain uniformly sized liposomes. Empty liposomes were prepared using the same protocol without Cur or BDMC.

\section{Particle characterization}

The particle size distribution of liposomes was determined using a dynamic light scattering instrument (LB-550; HORIBA Ltd., Kyoto, Japan). Liposomal dispersions were diluted with double-distilled water to ensure the light scattering intensity in the instrument's sensitivity range. The particle stability of the Cur- or BDMC-loaded liposomes (Cur-Lip and BDMC-Lip) was evaluated after storage at $4^{\circ} \mathrm{C}$ for 14 days and incubation with DMEM containing $10 \%$ bovine serum albumin at $37^{\circ} \mathrm{C}$ for 7 days. The measurements were performed in triplicate.

\section{Cell viability and proliferation assay}

7F2 osteoblast-like cells were seeded at a density of $10^{4}$ cells/well in 24-well plates for cell viability and proliferation studies. Cell medium was replaced with the medium containing test samples (empty liposomes, Cur-Lip, and BDMC-Lip) at various concentrations. After 1, 4, 7, and 14 days of incubation at $37^{\circ} \mathrm{C}$, the cell supernatants were removed and $200 \mu \mathrm{L}$ MTT (3-(4,5-Dimethylthiazol-2-yl)2,5-Diphenyltetrazolium Bromide) reagent $(100 \mu \mathrm{g} / \mathrm{mL})$ was added to each well for another 4 hours' incubation. To dissolve formazan crystals, $100 \mu \mathrm{L}$ dimethyl sulfoxide (DMSO) was added to each well and the absorbance was measured at $570 \mathrm{~nm}$ using an enzyme-linked immunosorbent assay (ELISA) reader (Tecan Trading AG, Tecan, Switzerland). All experiments were performed in quadruplicate, and the relative cell viability (\%) was expressed as a percentage relative to the untreated control cells.

\section{Entrapment efficiency}

After liposome formulation, the samples were spun down at $100,000 \mathrm{~g}$ for 30 minutes by Beckman ultracentrifuge. After centrifugation, the pellets were separated from the supernatant containing non-entrapped Cur or BDMC. The pellet was re-dissolved with ethanol and then the amount of Cur or BDMC in liposomes was measured by an ELISA reader at 427 or $419 \mathrm{~nm}$. The encapsulation efficiency (EE) was calculated using the following equation:

$$
\mathrm{EE}(\%)=\frac{\text { The amount of Cur/BDMC in liposomes }}{\text { Initial amount of Cur/BDMC for drug loading }} \times 100 \%
$$

For Cur, the sensitivity is $0.1 \mu \mathrm{g} / \mathrm{mL}$ and the linear range is $0.5-5 \mu \mathrm{g} / \mathrm{mL}$. For BDMC, the sensitivity is $0.1 \mu \mathrm{g} / \mathrm{mL}$ and the linear range is $0.5-5 \mu \mathrm{g} / \mathrm{mL}$.

\section{Fluorescence spectroscopy method for intracellular uptake of liposomes}

In order to study intracellular uptake of liposomes, $1 \mu \mathrm{L}$ of 1,1'-dioctadecyl-3,3,3',3'-tetramethylindocarbocyanine (DiI) (10 $\mathrm{mg} / \mathrm{mL}$ in ethanol) was added to the phospholipid solution. Then, DiI-labeled liposomes were prepared as described in the section "Liposome formulation". 7F2 cells were plated at a density of $2 \times 10^{4}$ cells $/$ dish in $6 \mathrm{~cm}$ dishes, and replaced with medium containing DiI-labeled liposomes after 24 hours. Thereafter, the cells were incubated with liposomes for a range of durations (0.5-8 hours), and fixed with $0.05 \%$ paraformaldehyde for 30 minutes. Samples were washed three times with phosphate-buffered saline (PBS), and stained with $5 \mu \mathrm{g} / \mathrm{mL} \mathrm{4}$, 6-diamidino-2-phenylindole (DAPI) for 5 minutes. After several washings with PBS, samples were imaged using a fluorescence microscope (Eclipse Ti-E; Nikon Instruments, Tokyo, Japan) with a charge-coupled device camera system (SPOT RT3; Diagnostic Instruments, Sterling Heights, MI, USA). To analyze liposome fluorescence in $7 F 2$ cells, fluorescence intensity was measured in over 100 individual cells, using $20 \times$ images obtained with equal acquisition parameters and Image $\mathbf{J}$ software (National Institutes of Health, MD, USA). 7F2 cells in the control group were cultured in medium containing DiI (DiI solution was added on the top of the medium without the mixing) for 24 hours.

\section{Nitrite measurement}

RAW 234.7 cells $\left(5 \times 10^{5}\right.$ cells/well $)$ were plated in 24 -well plates and incubated for 24 hours. The cells were stimulated with lipopolysaccharide (LPS) (500 ng/mL) for 24 hours and treated with curcuminoid-loaded liposomes (Cur-Lip and BDMC-Lip) at a concentration of 7-140 $\mu \mathrm{M}$. The nitric oxide (NO) released from the macrophages in the medium was assessed by measuring nitrite concentration. Samples $(100 \mu \mathrm{L})$ of the culture media were incubated with $100 \mu \mathrm{L}$ of Griess reagent at room temperature for 15 minutes in 96-well microplates. The absorbance at $550 \mathrm{~nm}$ was measured using an 
ELISA reader. Standard calibration curves were prepared using sodium nitrite to quantify nitrite expression from cells (the sensitivity is $2.5 \mu \mathrm{M}$ and the linear range is $20-100 \mu \mathrm{M}$ ).

\section{Osteoclast differentiation assay}

RAW 234.7 cells were cultured in 96-well plates at a density of 2,000 cells per well and incubated for 24 hours. The growth medium contained $50 \mathrm{ng} / \mathrm{mL}$ RANKL and $10 \mathrm{ng} / \mathrm{mL} \mathrm{LPS}$, and cells were treated with $70 \mu \mathrm{M}$ Cur-Lip or BDMC-Lip. The medium was replaced every 3 days. After 5 days' incubation, the samples were fixed with $10 \% \mathrm{w} / \mathrm{v}$ formaldehyde solution for 1 minute. Then, cells were stained with TRAP kit reagents as described in the manufacturer's instructions (Sigma-Aldrich Co.). The cells containing three or more nuclei were marked as TRAP-positive and the percentage of multinucleated cells in each sample was counted.

TRAP activity was determined by para-Nitropheny Phosphate (pNPP) according to the microplate assay method of Park et al with the following modifications. ${ }^{40}$ Cells were fixed with $10 \% \mathrm{w} / \mathrm{v}$ formaldehyde for 1 minute and then with an equal mixture of acetone and formalin for another minute. After they were completely dry, cells were incubated with $100 \mu \mathrm{L}$ phosphate substrate solution $(3.7 \mathrm{mM}$ pNPP and $10 \mathrm{mM}$ sodium tartrate in $50 \mathrm{mM}$ citrate butter, $\mathrm{pH} 4.6$ ) at $37^{\circ} \mathrm{C}$ for 10 minutes. Then, the enzyme reaction was stopped by the addition of $100 \mu \mathrm{L} 0.1 \mathrm{~N}$ sodium hydroxide solution and the absorbance of the resulting yellow-colored product was measured by an ELISA reader at a wavelength of $405 \mathrm{~nm}$.

\section{Cellular alkaline phosphatase activity}

7F2 osteoblast-like cells were cultured at a density of $10^{4}$ cells/well in 24-well plates in DMEM containing $10 \%$ FBS, $5 \mathrm{mM} \beta$-glycerol phosphate $(\beta-\mathrm{GP})$, and $50 \mu \mathrm{g} / \mathrm{mL}$ ascorbic acid with or without $70 \mu \mathrm{M}$ Cur-Lip or BDMC-Lip and incubated for $1,4,7$, and 14 days at $37^{\circ} \mathrm{C}$ in a $5 \% \mathrm{CO}_{2}$ atmosphere. The supernatants were removed and washed with PBS. Then, $1 \% \mathrm{v} / \mathrm{v}$ Triton solution was added to each well followed by incubation at $37^{\circ} \mathrm{C}$ for 10 minutes. After incubation, cell lysates were analyzed for alkaline phosphatase (ALP) using p-nitrophenyl phosphate (PNPP) as a substrate and diethanolamine buffer. PNPP solution $(200 \mathrm{~mL})$ was added into each well of the 96-well plate. The plate was incubated at room temperature for 30 minutes or until sufficient color developed. The stop solution $(50 \mu \mathrm{L} /$ well $)$ was added to stop reaction and the absorbance was measured using an ELISA reader at a wavelength of $405 \mathrm{~nm}$ (the sensitivity is $0.02 \mathrm{mM}$ and the linear range is $0.02-20 \mathrm{mM}$ ).

\section{Mineralization of the extracellular matrix}

7F2 osteoblast-like cells were seeded at a density of $10^{4}$ cells/well in $1 \mathrm{~mL}$ medium containing 10\% FBS, $5 \mathrm{mM}$ $\beta$-GP, and $50 \mu \mathrm{g} / \mathrm{mL}$ ascorbic acid and incubated for 7 , 14, and 21 days. Cur-Lip and BDMC-Lip $(70 \mu \mathrm{M})$ were added to the growth medium. The samples were washed with PBS, fixed with 75\% v/v ethanol, and dried at room temperature. After complete drying, the fixed cells were stained with $1 \%$ Alizarin Red S $(200 \mu \mathrm{L})$ for an hour. The images of cell morphology were taken with a microscope (Nikon TI-E) and charge-coupled device camera system (SPOT RT3). Four hundred microliters of 10\% w/v cetylpyridinium chloride solution was added to each well followed by shaking for 10 minutes to dissolve calcium. Finally, the absorbance was measured with an ELISA reader at a wavelength of $560 \mathrm{~nm}$. Each experiment was done in triplicate (the sensitivity is $0.25 \mathrm{mM}$ and the linear range is $0.25-2 \mathrm{mM}$ ).

\section{Quantitative real-time polymerase chain reaction}

Cells were seeded at a density of $2 \times 10^{5}$ cells in $6 \mathrm{~cm}$ dishes and incubated for 24 hours. RAW264.7 cells were treated with $70 \mu \mathrm{M}$ Cur-Lip or BDMC-Lip in the solutions of RANKL (50 ng/mL) and LPS (10 ng/mL) for 5 days. 7F2 cells were using $5 \mathrm{mM} \beta$-GP, and $50 \mu \mathrm{g} / \mathrm{mL}$ ascorbic acid to induce osteoblast mineralization, and stimulated inflammatory responses with IL-1 $\beta$ (10 ng/mL).

After treatment, total RNA was extracted using the TRIzol reagent (RiboZolTM; AMRESCO, OH, USA) following the protocol in the manufacturer's instructions. For reverse transcription, $1 \mu \mathrm{g}$ of the total RNA was converted to first-strand cDNA using a reverse transcription kit (Promega Corporation, Fitchburg, WI, USA). The resulting cDNA (equivalent to $20 \mathrm{ng}$ ) was used in a StepOnePlus ${ }^{\mathrm{TM}}$ Real-Time PCR System using Fast Start DNA Master-PLUS SYBR Green I (Thermo Fisher Scientific). The designed primers are shown in Table 1; all primers used nucleotide sequences present in the PrimerBank database. Each sample was corrected using the mean cycle threshold (CT) value for GAPDH. Relative gene expression was analyzed using the $\Delta \mathrm{C}_{\mathrm{T}}$ method and expressed as fold change $\left(2_{\mathrm{T}}^{-\Delta \mathrm{CC}}\right)$.

\section{Statistical analysis}

The experiments were repeated at least twice with similar results, and the values were expressed as means \pm standard deviations. Data analysis was performed by paired $t$-test using the commercial software MINITAB. Differences were considered to be statistically significant at $P<0.05$. 
Table I Sequences of primers used in real-time polymerase chain reaction experiment

\begin{tabular}{lll}
\hline Target & Forward $\left(\mathbf{5}^{\prime} \mathbf{-} \mathbf{3}^{\prime} \mathbf{)}\right.$ & Reverse $\mathbf{( 5 ^ { \prime } - \mathbf { 3 } ^ { \prime } )}$ \\
\hline GAPDH & CATGAGAAGTATGACAACAGCCT & AGTCCTTCCACGATACCAAAGT \\
Cathepsin K & ATAACGCCACGGCAAAGG & TTCTCGTTCCCCACAGGAAT \\
TRAP & GCAACATCCCCTGGTATGTG & GCAAACGGTAGTAAGGGCTG \\
COX-2 & CAGCCAGGCAGCAAATCC & ACATTCCCCACGGTTTTGAC \\
MMP-3 & GGCCTGGAACAGTCTTGGC & TGTCCATCGTTCATCATCGTCA \\
RANKL & CGCTCTGTTCCTGTACTTTCG & GAGTCCTGCAAATCTGCGTT \\
OPG & CCTTGCCCTGACCACTCTTAT & CACACACTCGGTTGTGGGT \\
\hline
\end{tabular}

\section{Results}

\section{In vitro characterization of liposomes}

The effect of lipid composition on particle sizes and stability of liposomes formulated from different ratios of SPC and cholesterol was monitored at $4^{\circ} \mathrm{C}$ for a period of 2 weeks (Figure 1A). The mean size of liposomes is an important requisite that can influence the drug carrier's biological effectiveness. ${ }^{41}$ In order to produce smaller liposomes, extrusion

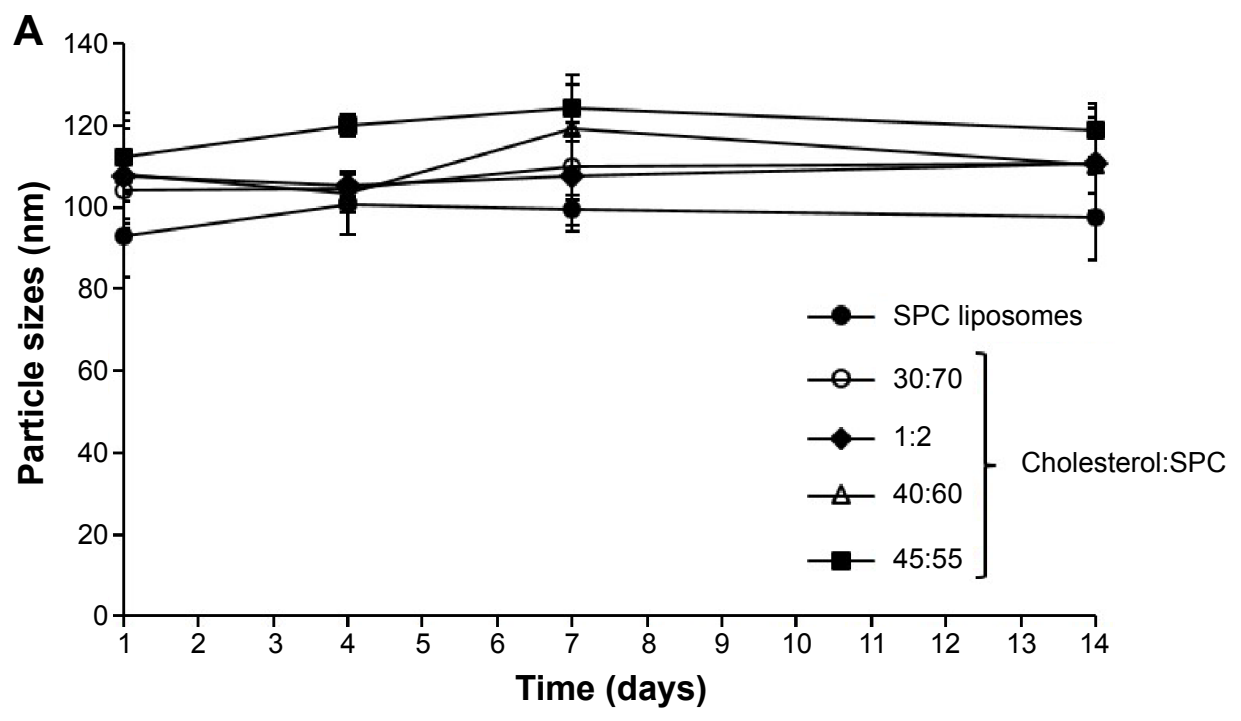

B

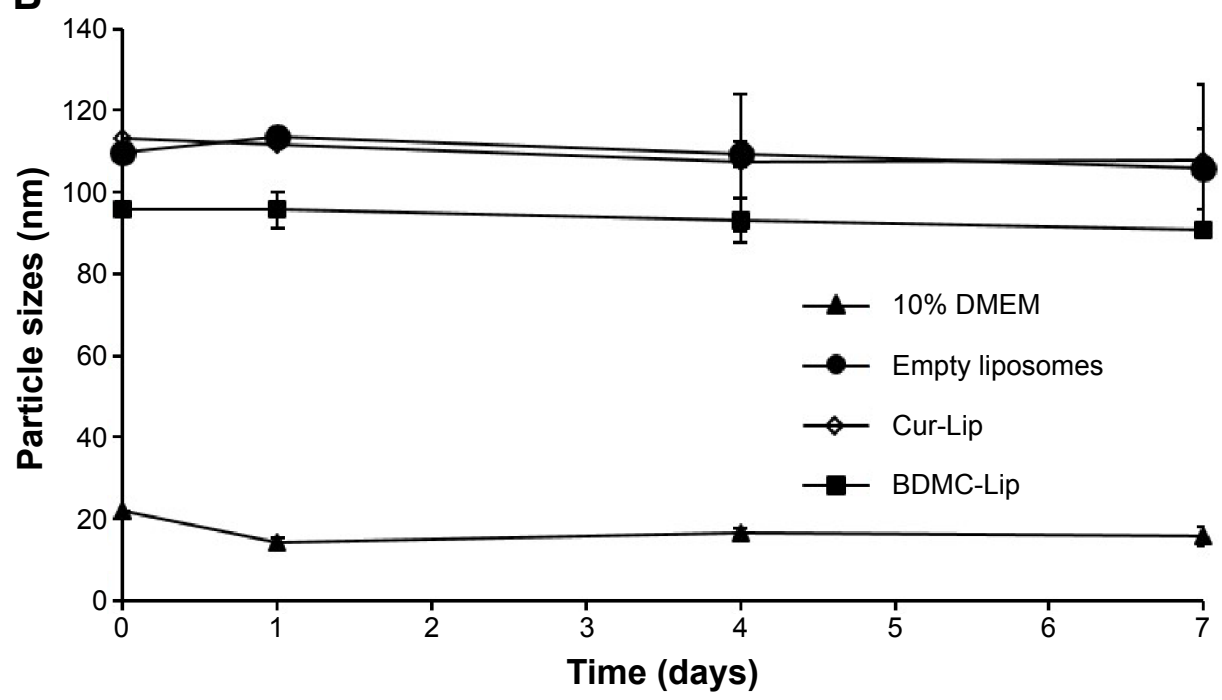

Figure I Particle sizes of liposome formulations.

Notes: (A) The stability of liposomes prepared with different compositions of SPC and cholesterol after I, 4, 7, and I4 days of incubation. (B) The stability of empty liposomes, Cur-Lip, and BDMC-Lip in DMEM with $10 \%$ fetal bovine serum after I, 4, and 7 days of incubation.

Abbreviations: BDMC-Lip, bisdemethoxycurcumin-loaded liposomes; Cur-Lip, curcumin-loaded liposomes; DMEM, Dulbecco's Modified Eagle's Medium; SPC, soybean phosphatidylcholine. 
through polycarbonate filters was carried out after hydration. Without extrusion, the mean diameter of liposomes was greater than $900 \mathrm{~nm}$ and the standard deviation was around $200 \mathrm{~nm}$, showing the presence of large vesicles with a very large size distribution (Table 2). The extrusion procedure provided small multilamellar vesicles with mean diameters ranging from 95-120 $\mathrm{nm}$. This procedure also allowed the formation of liposome suspensions with a very narrow size distribution having a standard deviation value less than $10 \mathrm{~nm}$. At $4^{\circ} \mathrm{C}$, these liposome systems displayed similar stability profiles over a 2 -week period. In contrast, these liposomes are more stable than vesicles without extrusion (data not shown). Three formulations of liposomes were prepared in this study: empty liposomes, Cur-Lip, and BDMC-Lip. The stability of liposomes in vivo can be affected by interactions with lipoproteins and other proteins in the blood. The stability tests of liposomes in DMEM containing 10\% bovine serum albumin showed less than $10 \%$ change in the particle size distribution in the three types of liposomal formulations (Figure 1B). As reported in Table 2, the curcuminoids showed high entrapment efficiency due to their lipophilic nature. After extrusion, the entrapment efficiency was decreased, possibly due to particle size reduction. Therefore, Cur-Lip and BDMC-Lip achieved maximum encapsulation efficiencies of $69.5 \%$ and $71.4 \%$ after extrusion.

\section{Effect of liposomal formulations on cell viability, delivery efficiency, and $\mathrm{NO}$ production}

The in vitro cytotoxic potential of liposomes formulated with various lipid compositions was assessed in mouse osteoblastic cell line 7F2, using the MTT assay. Lipid compositions in a range of molar ratios of cholesterol to SPC (30:70, 1:2, 40:60, and 45:55) resulted in slightly higher viability loss than SPC only (Figure 2A). Therefore, cholesterol was omitted in favor of SPC in liposomal preparation. Next, a dose-response study of the curcuminoid-loaded liposomes evaluated cell

Table 2 Encapsulation parameters of the liposomal formulations

\begin{tabular}{|c|c|c|c|c|}
\hline \multirow[t]{2}{*}{ Liposomes } & \multicolumn{2}{|l|}{$\begin{array}{l}\text { Particle } \\
\text { size }(\mathrm{nm})\end{array}$} & \multicolumn{2}{|c|}{$\begin{array}{l}\text { Entrapment } \\
\text { efficiency (\%) }\end{array}$} \\
\hline & Pre-extrusion & $\begin{array}{l}\text { Pro- } \\
\text { extrusion }\end{array}$ & $\begin{array}{l}\text { Pre- } \\
\text { extrusion }\end{array}$ & $\begin{array}{l}\text { Pro- } \\
\text { extrusion }\end{array}$ \\
\hline Empty & $959.6 \pm 204.3$ & $99.7 \pm 4.2$ & - & - \\
\hline Cur-Lip & $1,110.0 \pm 149.0$ & $110.0 \pm 4.8$ & $92.5 \pm 4.2$ & $69.5 \pm 1.4$ \\
\hline BDMC-Lip & $1,097.9 \pm 135.4$ & $110.8 \pm 8.5$ & $89.4 \pm 0.7$ & $71.04 \pm 7.4$ \\
\hline
\end{tabular}

Note: Values represent the mean \pm standard deviation for at least three experiments.

Abbreviations: BDMC-Lip, bisdemethoxycurcumin-loaded liposomes; Cur-Lip, curcumin-loaded liposomes. viability of 7F2 osteoblastic cells with MTT. As a whole, the viability loss is dose-related. When the curcuminoid concentration in liposomes was increased to $140 \mu \mathrm{M}$, a significant viability loss was noted (Figure 2B). Furthermore, Cur-Lip and BDMC-Lip showed less cytotoxicity than free drugs in DMSO (70 $\mu \mathrm{M})$ (Figure 2C).

To study the cellular uptake of curcuminoid-loaded liposomes, DiI-loaded liposomes (red fluorescence) and DAPI-stained cells (blue fluorescence) were used to evaluate the delivery efficiency of liposomes. Cells were harvested at different time intervals and cellular uptake was observed by fluorescence microscopy (Figure 3A). The results showed that the uptake processes were started at 30 minutes after exposure and reached their maximum after 2 hours (Figure 3B).

The anti-inflammatory activity of curcuminoid-loaded liposomes in LPS-stimulated RAW264.7 macrophages was investigated by assessing the inhibition of NO. The amount of nitrite measured by the Griess reagent correlated directly to the NO production by RAW264.7 macrophages. When LPS-stimulated RAW264.7 macrophages were incubated with empty and curcuminoid-loaded liposomes, NO production in LPS-stimulated RAW264.7 macrophages was significantly decreased (Figure 4A). Curcuminoid-loaded liposomes inhibited NO production in a dose-dependent manner (in a range of 7-70 $\mu \mathrm{M}$ ) (Figure 4B), but there was no significant difference in the reduction of $\mathrm{NO}$ production between Cur-Lip and BDMC-Lip.

\section{Inhibitory effects of curcuminoid-loaded liposomes on osteoclast differentiation in RAW264.7 macrophages}

To examine the potential of curcuminoid-loaded liposomes in osteoclast differentiation, RAW264.7 macrophages were incubated in the osteoclastogenic medium containing $10 \mathrm{ng} / \mathrm{mL}$ LPS and $50 \mathrm{ng} / \mathrm{mL}$ RANKL for 5 days, which led to a profound differentiation of the monocyticmacrophagic cell line into multinucleated TRAP-positive cells. The percentage of differentiated osteoclasts was significantly increased with the addition of LPS and RANKL, and the differentiated cells were larger than normal. The cells treated with Cur-Lip or BDMC-Lip showed reductions in cell size and the percentage of multinucleation (Figure 5A). In addition, when RAW264.7 macrophages were toward osteoclast differentiation and maturation, osteoclasts would express high TRAP activity for removing mineralized deposition. Therefore, osteoclast differentiation can be evaluated by measuring TRAP activity. As shown in Figure 5B, RAW264.7 macrophages with 
A

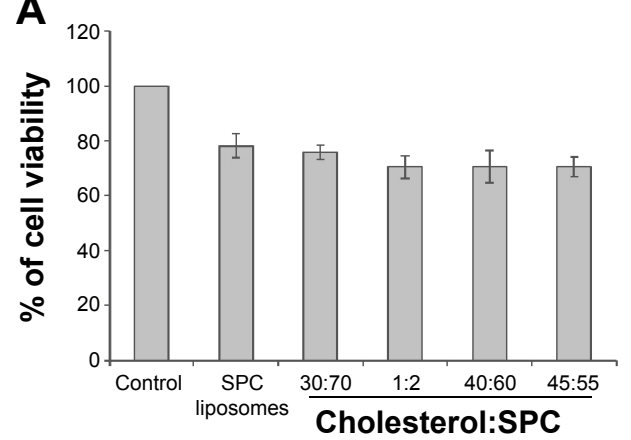

B

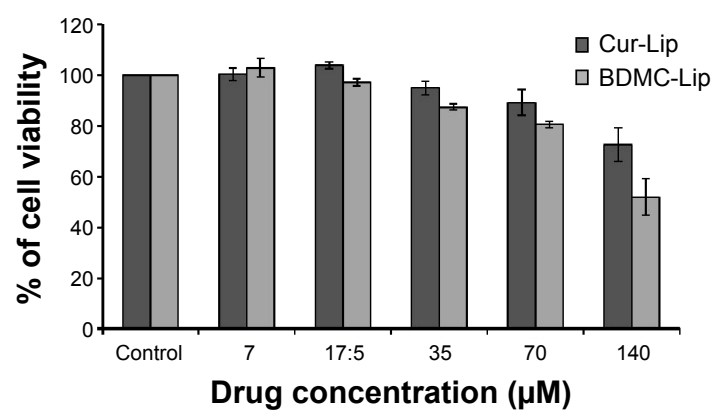

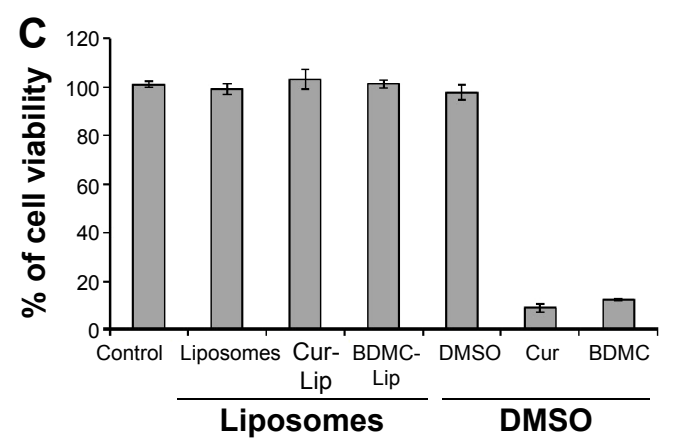

Figure 2 The effect of curcuminoid-loaded liposomes on cell viability.

Notes: 7F2 osteoblastic cells were incubated with different test materials for 24 hours and then examined by MTT method. (A) Influence of lipid composition of empty liposomes. (B) Effect of concentrations of Cur-Lip and BDMC-Lip. (C) The survival rate of 7F2 osteoblastic cells after treatment with $70 \mu M$ curcuminoid-loaded liposomes or Cur or BDMC in DMSO. Data are expressed as the mean of percent cell viability compared to control after exposure for 24 hours \pm standard deviation ( $\mathrm{n}=3-6$ ). Abbreviations: BDMC, bisdemethoxycurcumin; BDMC-Lip, BDMC-loaded liposomes; Cur, curcumin; Cur-Lip, Cur-loaded liposomes; DMSO, dimethyl sulfoxide; MTT, 3-(4,5-dimethylthiazol-2-yl)-2,5-diphenyltetrazolium bromide; SPC, soybean phosphatidylcholine.

the treatment of LPS and RANKL presented higher TRAP activity. On the other hand, Cur-Lip and BDMC-Lip significantly reduced the TRAP activity, and that is roughly equivalent to control. With a view to finding out whether or not Cur-Lip and BDMC-Lip caused the suppression of osteoclastogenic differentiation due to altering cathepsin $\mathrm{K}$ and TRAP expression, RAW264.7 macrophages were assayed for the gene expression of cathepsin $\mathrm{K}$ and TRAP, which were previously known to be able to regulate osteoclast differentiation. All liposomal formulations revealed significant inhibition on RANKL-induced cathepsin $\mathrm{K}$ and TRAP gene expression (Figure 5C and D). Therefore, Cur-Lip and BDMC-Lip can inhibit RANKL-induced osteoclast differentiation and resorption activity (TRAP activity) through the suppression of cathepsin K and TRAP expression.

\section{Effect on 7F2 osteoblasts of curcuminoid- loaded liposomes}

The effect of curcuminoid-loaded liposomes on osteoblast proliferation was measured using the MTT assay (Figure 6A). The exposure of 7F2 osteoblastic cells to liposomes decreased cell number by approximately $10 \%$ after 7 days' incubation, as compared with cells in mineralizing medium (MM) $(50 \mu \mathrm{g} / \mathrm{mL}$ ascorbic acid and $5 \mathrm{mM} \beta$-GP).
Furthermore, curcuminoid-loaded liposomes showed no marked inhibition on cell proliferation in a time-dependent manner.

Figure 6B presents the ALP activity of 7F2 osteoblastic cells in MM with or without the addition of liposomes (empty liposomes, Cur-Lip, and BDMC-Lip) after the number of days indicated. As expected, ALP activity was constantly increased with culture time in control and MM, but this increase was significantly blunted by liposome treatment. Therefore, empty and curcuminoid-loaded liposomes showed significant inhibition of ALP activity. It is worth noting that cells cultured in the medium containing Cur-Lip expressed higher levels of ALP activity than those with empty liposomes or BDMC-Lip.

The effects of curcuminoid-loaded liposomes on osteoblast mineralization was investigated after 7, 14, and 21 days of incubation using Alizarin Red S staining, which identifies calcium content within the bone matrix. Increased mineralization relative to controls was observed in cultures with $\mathrm{MM}$, but mineral deposition was reduced in all groups of liposome-treated osteoblasts (Figure 6C and D). Under microscope observation, the numbers of mineralized nodules (red) were clearly lower on 7F2 osteoblastic cells treated with empty liposomes and BDMC-Lip on incubation day 21 


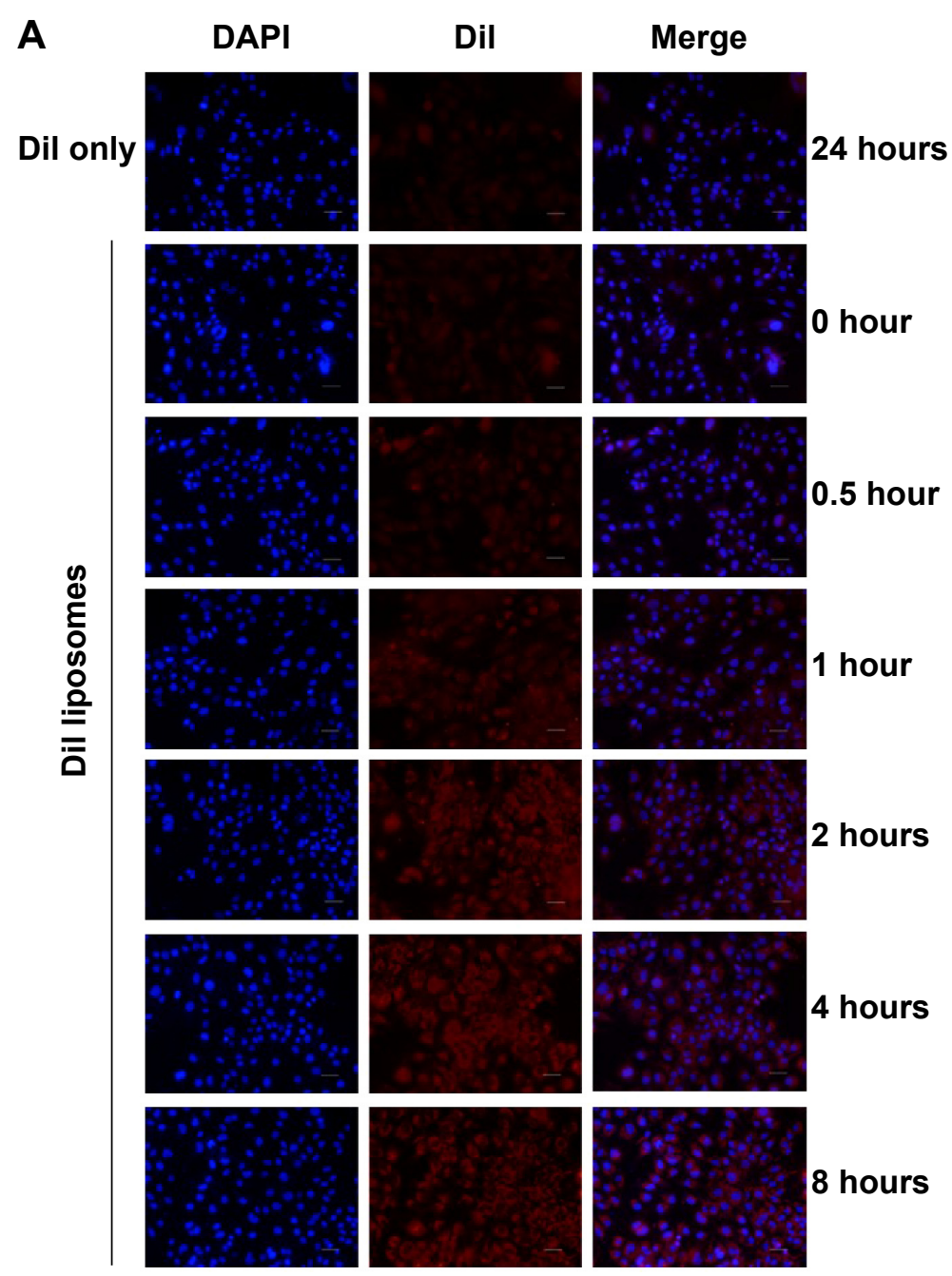

B

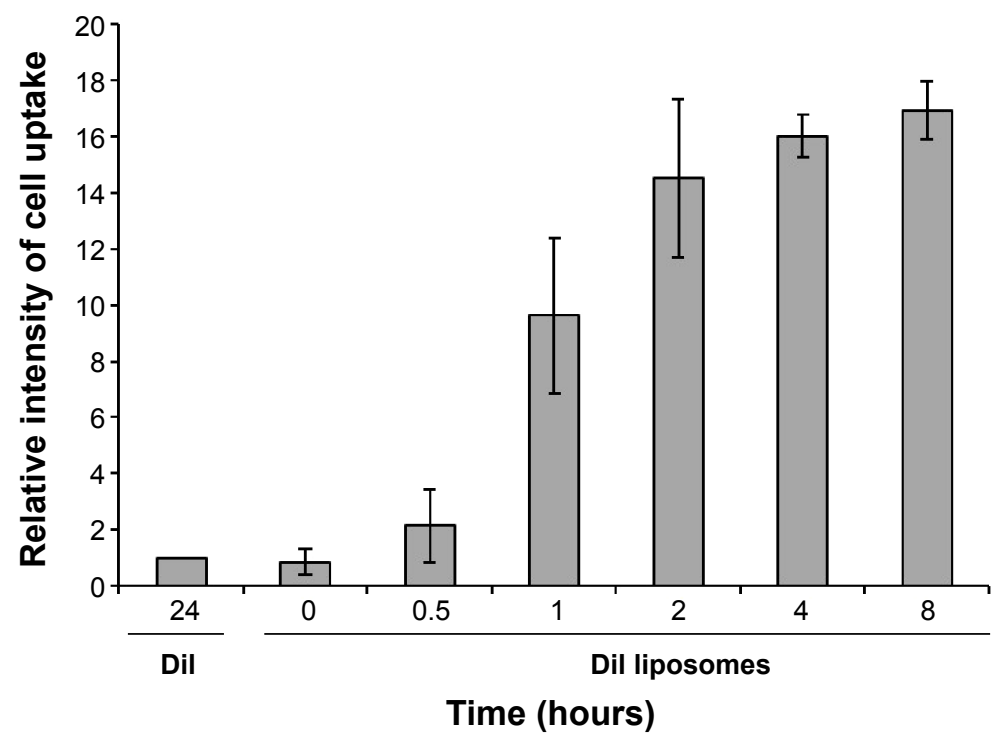

Figure 3 Cell uptake of liposomes on 7F2 osteoblastic cells.

Notes: (A) The cells were incubated for different time intervals and cell uptake (red fluorescence) was determined by fluorescence microscopy. Cells were also stained with DAPI (blue fluorescence). (B) Quantification of cellar uptake. The results are expressed as the mean of percent cell uptake compared to Dil only \pm standard deviation $(n=3-6)$.

Abbreviations: DAPI, 4',6-diamidino-2-phenylindole; Dil, I,I'-dioctadecyl-3,3,3',3'-tetramethylindocarbocyanine. 
A

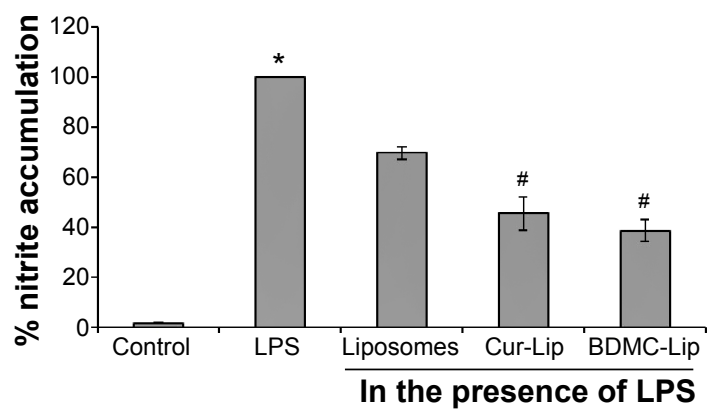

B

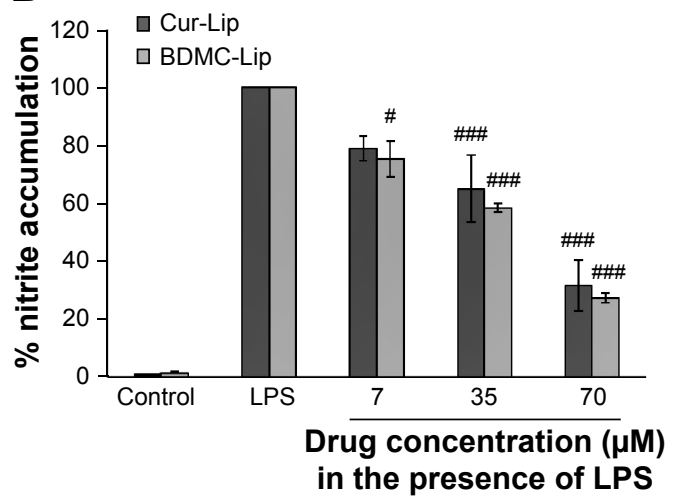

Figure 4 Effects of curcuminoid-loaded liposomes on NO production in LPS (500 ng/mL)-induced RAW264.7 macrophages for 24 hours.

Notes: (A) Empty and curcuminoid-loaded liposomes inhibited LPS-induced NO production. (B) Cur-Lip and BDMC-Lip dose-dependently inhibited LPS-induced NO production. Cells of the control group were not induced with LPS. The total nitrite produced by the cells of the LPS group (with the treatment of 500 ng/mL LPS) is expressed as $100 \%$. Results are expressed as percentage with mean \pm standard deviation $(n=3-6)$. $* P<0.005$ with respect to control and ${ }^{\#} P<0.05$ with respect to liposome $(\mathbf{A})$. ${ }^{\#}<0.05$ and $P<0.005$ with respect to LPS (B).

Abbreviations: BDMC-Lip, bisdemethoxycurcumin-loaded liposomes; Cur-Lip, curcumin-loaded liposomes; LPS, lipopolysaccharide; NO, nitric oxide.

(Figure 6C), but Cur-Lip still revealed high mineralized nodule formation. Similar patterns were observed in ALP activity.

OPG and RANKL are key factors in regulating bone metabolism; the ratio of OPG/RANKL is considered to better reflect environmental signals in OA patients than the levels of each of these factors individually, in which a high ratio level is indicative of bone formation promotion while a low level favors bone resorption. ${ }^{42}$ Our results indicated that Cur-Lip and BDMC-Lip increased the OPG/RANKL ratio significantly in 7F2 osteoblastic cells (Figure 6E). In contrast, the relative ratio of OPG/RANKL was lower in MM with or without the addition of empty liposomes than in control. Therefore, Cur-Lip can inhibit osteoclast differentiation through the increase of OPG/RANKL ratio, and retain osteoblast differentiation functions better than BDMC-Lip.

\section{Curcuminoid-loaded liposomes protective osteoblast when stimulated with il-I $\beta$}

$7 F 2$ osteoblastic cells responded to IL- $1 \beta$ stimulation by upregulated expression of proinflammatory mediators, cyclooxygenase 2 (COX-2) and MMP-3 (Figure 7A and B). Cells treated with empty or curcuminoid-loaded liposomes can suppress IL- $1 \beta$-induced COX-2 and MMP-3 production (Figure 7C and D). This inhibitory effect was not significantly different between empty and curcuminoid-loaded liposomes at a concentration of $70 \mu \mathrm{M}$. These data revealed a tendency toward decrease in COX-2 and MMP-3 mRNA levels with respect to basal expression, suggesting that liposomal
A
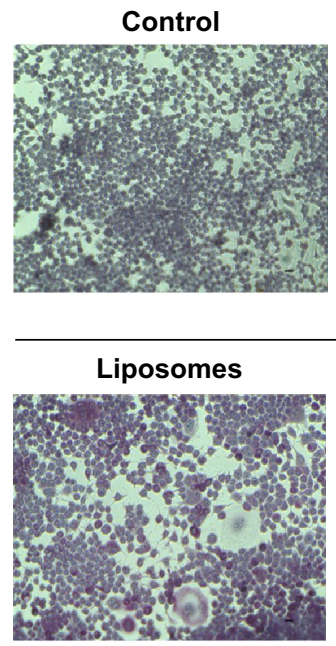

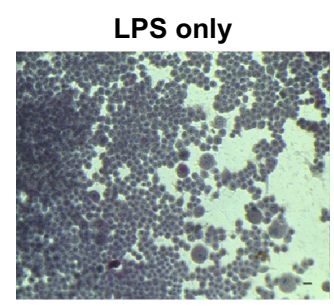

LPS + RANKL

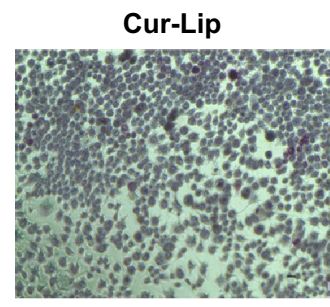

RANKL only

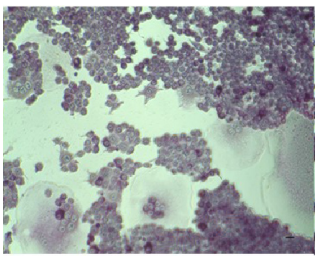

BDMC-Lip

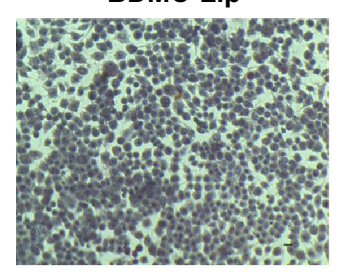

LPS + RANKL

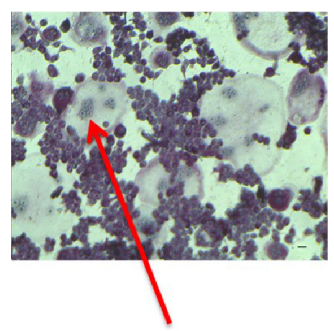

Multinucleated ( $\geq 3$ nuclei) cells

Figure 5 (Continued) 


\section{B}

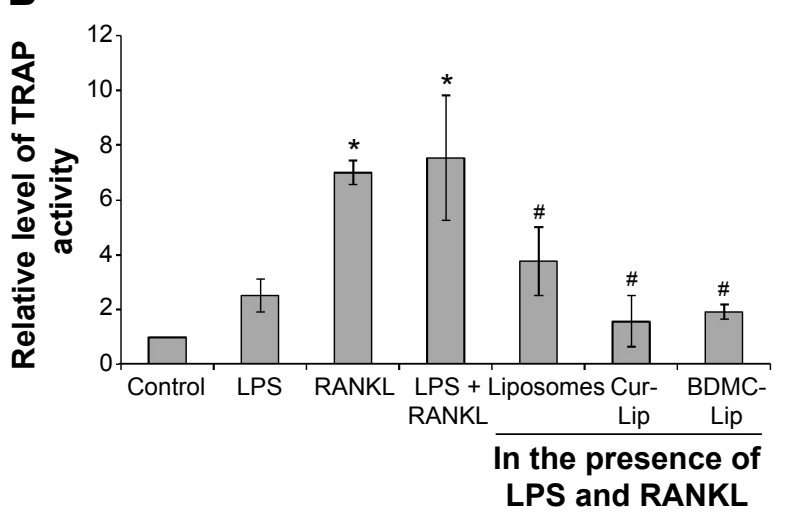

C

D

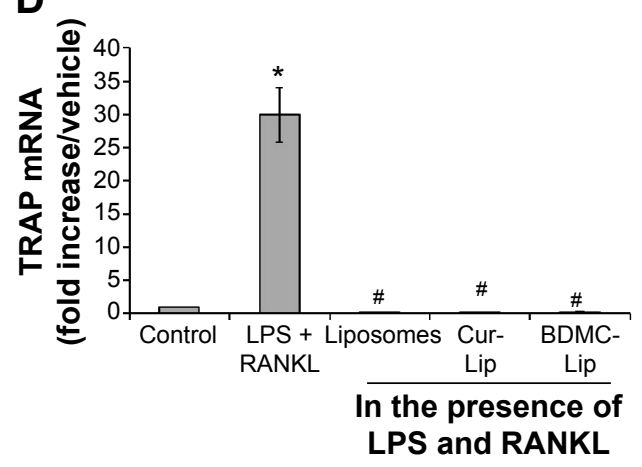

Figure 5 Curcuminoid-loaded liposomes $(70 \mu \mathrm{M})$ suppress osteoclastogenesis in $10 \mathrm{ng} / \mathrm{mL}$ LPS- and 50 ng/mL RANKL-induced RAW264.7 macrophages after 5 days of cultivation.

Notes: (A) The effect of curcuminoid-loaded liposomes on TRAP staining of RAW264.7 macrophages. Photographs are of representative osteoclast cultures under bright field ( $\times 200$ magnification). Osteoclasts are shown by red arrow in the LPS- and RANKL-treated culture. Scale bars represent $10 \mu \mathrm{m}$. (B) Empty and curcuminoid-loaded liposomes inhibit TRAP activity on LPS- and RANKL-induced RAW264.7 macrophages. Data are expressed as the relative ratio of TRAP activity measured in cells derived from each experimental model in comparison with control. The images are representative of two independent experiments (four wells per condition were counted for each experiment). (C and D) LPS and RANKL induced cathepsin K and TRAP mRNA expression and Cur-Lip and BDMC-Lip inhibited TRAP and cathepsin K activation. Quantitation of cathepsin K and TRAP mRNA expression are relative to GAPDH. Data are presented as the means \pm standard deviation ( $\mathrm{n}=3-6)$. ${ }^{*}>0.005$ with respect to control and $\# P<0.05$ with respect to liposome.

Abbreviations: BDMC-Lip, bisdemethoxycurcumin-loaded liposomes; Cur-Lip, curcumin-loaded liposomes; LPS, lipopolysaccharide; RANKL, receptor activator of nuclear factor $\kappa B$ ligand; TRAP, tartrate-resistant acid phosphatase.

formulations can suppress IL-1 $\beta$-induced inflammatory responses on 7F2 osteoblastic cells. To study the influence of curcuminoid-loaded liposomes on osteoclastogenesisregulatory biomarkers on 7F2 osteoblastic cells, we further examined the mRNA expression of OPG and RANKL. As shown in Figure 7E, inflammatory responses reduced the OPG/RANKL ratio in 7F2 osteoblastic cells, indicative of a possible increase in bone remodeling/resorption capacity. With the addition of liposomes, OPG/RANKL ratios were expressed highly in 7F2 osteoblastic cells in the presence of IL-1 $\beta$ stimulation (Figure 7E). Of note, only Cur-Lip expressed a high OPG/RANKL ratio in the absence of IL-1 $\beta$ stimulation (Figure 6E). Therefore, these findings support that curcuminoid-loaded liposomes can downregulate IL-1 $\beta$-induced MMP-3 and COX-2 expression and suppress osteoclastogenesis through the increase of the OPG/RANKL ratio.

\section{Discussion}

In recent years, several novel delivery systems including polymeric implantable delivery systems have been proposed to improve drug bioavailability. ${ }^{34-37}$ In this study, we optimized liposomal formulation by using SPC (Phospholipon 90G) and cholesterol to develop liposomal delivery systems of curcuminoids, because soy phospholipids have been successfully used to improve the poor solubility and consequently low bioavailability of Cur. ${ }^{38,39}$ According to the literature, SPC liposomes can successfully increase the bioavailability of Cur in a rat model system, and liposomes composed with SPC showed better entrapment efficiency than other lipids. ${ }^{42}$ Our results have also shown high entrapment efficiencies of Cur and BDMC (>70\%) and significant stability over 14 days. In cytotoxicity study, the concentration of Cur or BDMC in DMSO $(70 \mu \mathrm{M})$ used to treat cells was twofold lower than the concentration added 
A

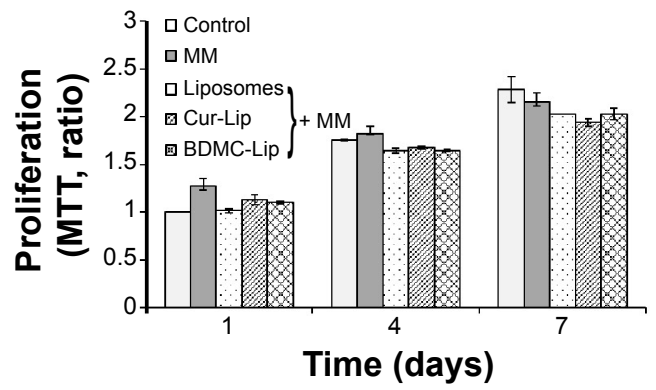

B

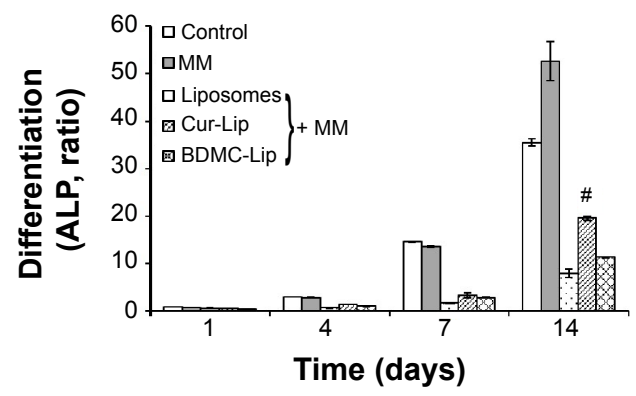

D

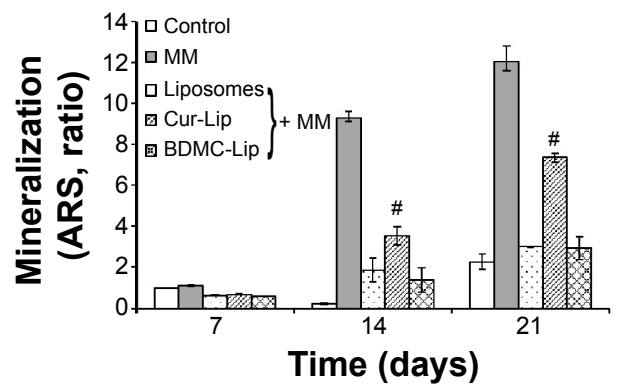

$\mathbf{E}$

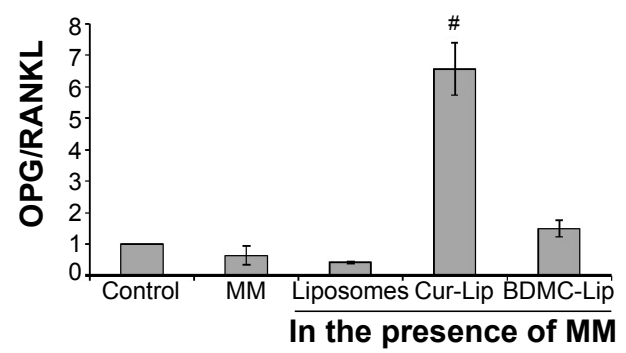

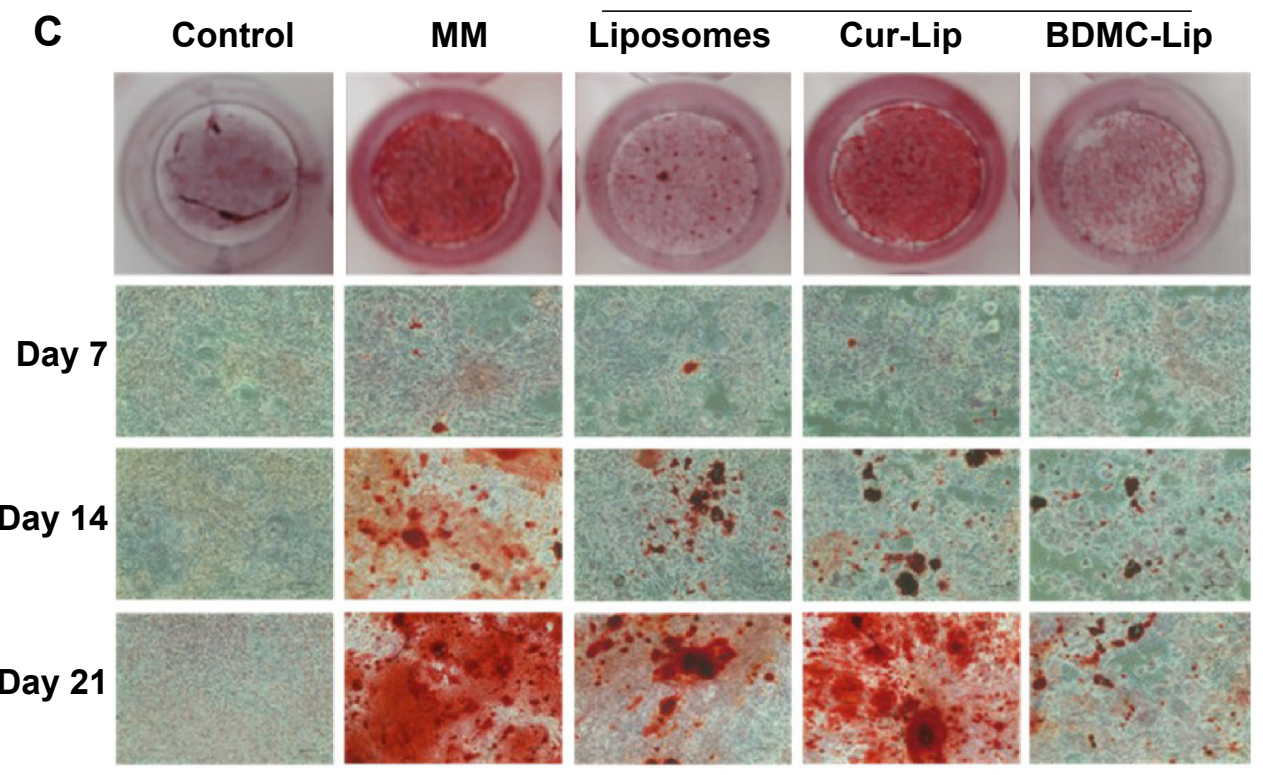

Figure 6 Effects of curcuminoid-loaded liposomes $(70 \mu \mathrm{M})$ on osteoblast proliferation, differentiation, and mineralization.

Notes: 7F2 osteoblastic cells were cultured in MM ( $50 \mu \mathrm{g} / \mathrm{mL}$ ascorbic acid and $5 \mathrm{mM} \beta$-GP) to induce osteoblast differentiation and mineralization. (A) The effects of liposomal formulations on cell proliferation. Data are expressed as the mean of percent cell viability compared to the control after exposure \pm standard deviation ( $n=3-6)$. (B) The effects of liposomal formulations on ALP assay. After I, 4, 7, and I4 days' incubation in control or MM in the presence or absence of curcuminoid-loaded liposomes, ALP activity was measured by the manual instruction. The results of ALP activity in the control, MM, and liposomal groups are displayed in columns with standard deviation values. ALP activity decreased significantly in the 7F2 osteoblasts of liposomal groups compared with the activity in the MM group. All samples were performed in triplicate. (C) Histochemical staining of mineral deposition. 7F2 osteoblastic cells were cultured in control or MM in the presence or absence of liposomes for 7, 14, and 2I days and mineral deposition was visualized by ARS staining ( $\times 100$ magnification, $n=3$ ). Red staining represents mineral deposition. (D) Quantification of mineralization. ARS stain was quatified by cetylpyridinium chloride extraction method. 7F2 osteoblastic cells treated with liposomes decreased the ARS accumulation in comparison with the MM group. Data are representative of two independent experiments. Photographs are of representative osteoblast cultures under bright field. (E) The effects of curcuminoid-loaded liposomes on the OPG/RANKL ratio of 7F2 osteoblastic cells. 7F2 osteoblastic cells were treated with MM in the presence or absence of liposomes for 3 days, following which, RNA was extracted and real time RT-PCR analysis was performed to examine the gene expression of OPG and RANKL (relative to GADPH). The data are presented as the means \pm standard error. ${ }^{\# P}<0.005$, Student's t-test relative to the liposome.

Abbreviations: ARS, Alizarin Red S; BDMC-Lip, bisdemethoxycurcumin-loaded liposomes; Cur-Lip, curcumin-loaded liposomes; MM, mineralizing medium; MTT, 3-(4,5-dimethylthiazol-2-yl)-2,5-diphenyltetrazolium bromide; ALP, alkaline phosphatase; RANKL, receptor activator of nuclear factor KB ligand; OPG, osteoprotegerin; ALP, alkaline phosphatase; PCR, polymerase chain reaction. 
A
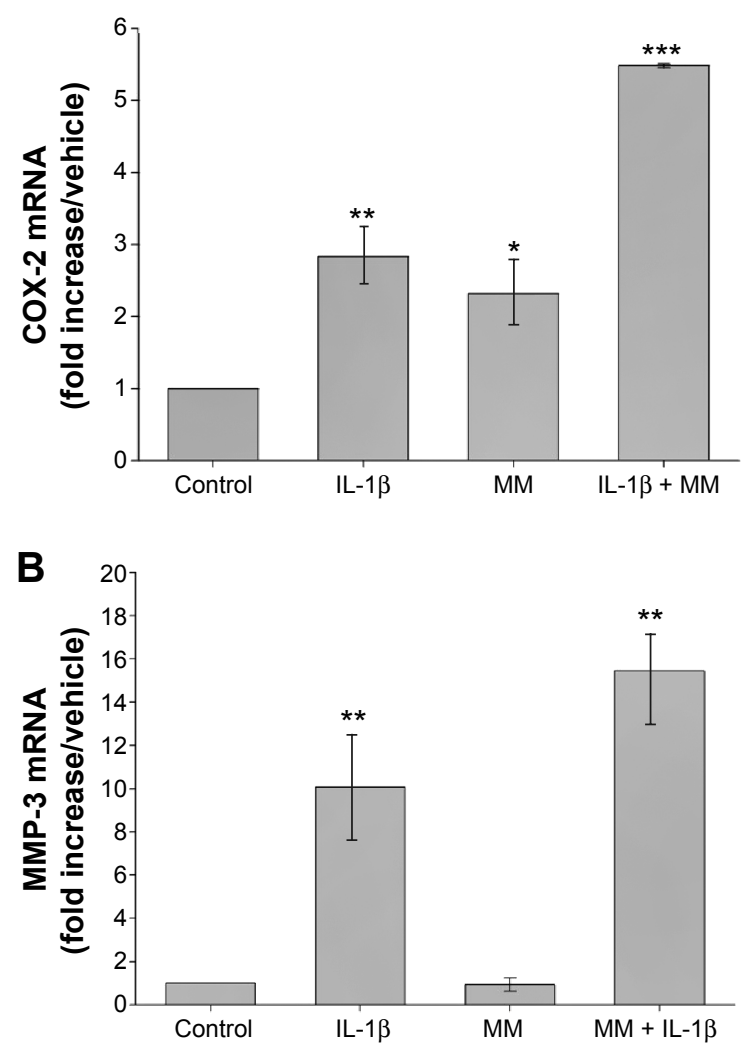

D

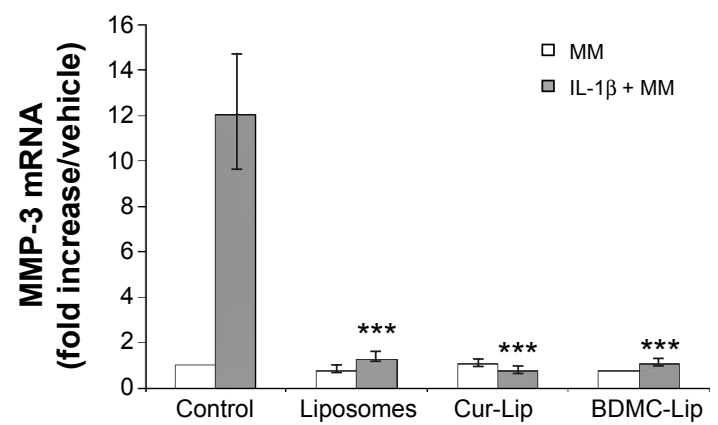

E

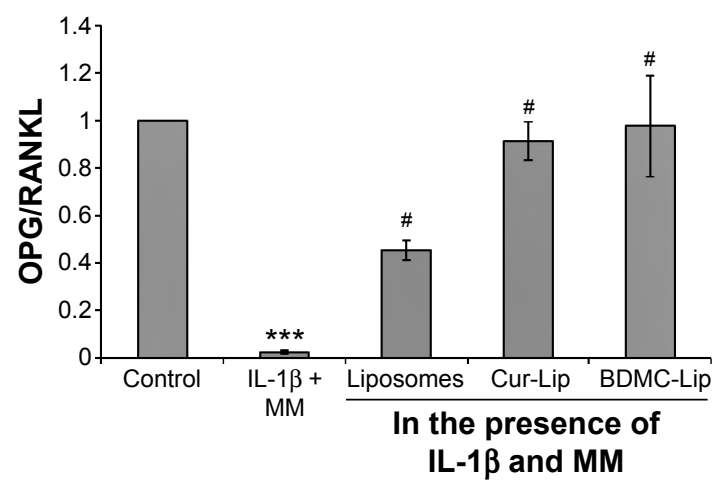

C

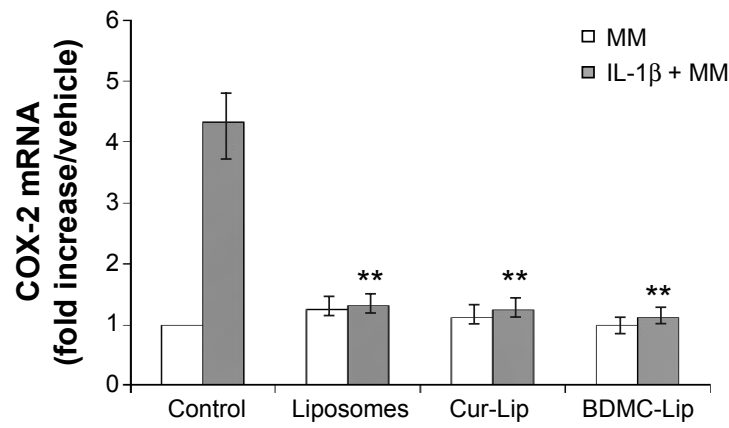

Figure 7 Effects of curcuminoid-loaded liposomes $(70 \mu \mathrm{M})$ on the expression of proinflammatory mediators (COX-2 and MMP-3), and OPG/RANKL ratio of 7F2 osteoblastic cells in the presence or absence of $10 \mathrm{ng} / \mathrm{mL} \mathrm{IL}-\mathrm{I} \beta$ stimulation.

Notes: 7F2 osteoblastic cells were treated with $50 \mu \mathrm{g} / \mathrm{mL}$ ascorbic acid and $5 \mathrm{mM} \beta$-GP to induce osteoblast differentiation and mineralization (MM) and inflamed by $10 \mathrm{ng} / \mathrm{mL}$ IL-I $\beta$. (A and B) The influence of IL-I $\beta$ on COX-2 mRNA and MMP-3 mRNA expression of 7F2 osteoblastic cells. (C and D) Effects of curcuminoid-loaded liposomes on mRNA expression of COX-2 and MMP-3 in 7F2 osteoblastic cells. (E) Effects of curcuminoid-loaded liposomes on OPG/RANKL ratio of 7F2 osteoblastic cells in the presence of IL-I $\beta$ and MM. Real-time polymerase chain reaction analysis was performed in 7F2 osteoblastic cells incubated with MM and IL-I $\beta$ for 4 hours. Data represent mean \pm standard error $(n=3-6)$. $* P<0.05$, $* * P<0.01$, and $* * * P<0.005$ with respect to control and $* P<0.005$ with respect to liposome.

Abbreviations: BDMC-Lip, bisdemethoxycurcumin-loaded liposomes; COX-2, cyclooxygenase 2; Cur-Lip, curcumin-loaded liposomes; MM, mineralizing medium; MMP-3, matrix metalloproteinase-3; RANKL, receptor activator of nuclear factor $\kappa B$ ligand; OPG, osteoprotegerin; IL, interleukin.

into liposomes $(140 \mu \mathrm{M})$. However, 7F2 osteoblasts treated with Cur-Lip or BDMC-Lip showed a sixfold higher survival rate than those treated with Cur or BDMC in DMSO. Moreover, SPC liposomes displayed 20-fold $(P<0.001)$ higher intracellular accumulations than the free drug on 7F2 osteoblastic cells. As a result of this, liposome formulation could reduce cytotoxicity and increase cellular uptake of curcuminoids.

The results of Tocharus et al indicated that Cur, DMC, and BDMC can all inhibit NO production in LPS-activated HAPI microglial cells. ${ }^{22}$ In addition, Cur was found to be the most active metabolite of curcuminoid in suppressing 
activation of TNF-induced NF- $\mathrm{KB}$ (Cur $>\mathrm{DMC}>$ BDMC). ${ }^{32}$ Recently, Jung et al showed that Cur had strong NO inhibitory potential through the inhibition of JNK, p38, and NF- $\mathrm{KB}$ signaling pathways. ${ }^{43}$ Although the ability of curcuminoids to inhibit the inflammatory response and suppress the osteoclastogenesis is established, how these responses are modulated by curcuminoid-loaded liposomes is not known. Our results indicate that Cur- and BDMCloaded liposomes can inhibit NO production in RAW264.7 macrophages and prevent osteoclast differentiation through the downregulation of cathepsin $\mathrm{K}$ and TRAP expression. We also found that Cur-Lip and BDMC-Lip presented high OPG/RANKL ratios in IL-1 $\beta$-induced 7F2 osteoblastic cells. The high level of OPG/RANKL ratio indicated the favorite of OPG-RANKL interaction and the prevention of its binding to RANK with a consequent decrease in osteoclast activation. Therefore, the inhibitory role of curcuminoid-loaded liposomes on RANKL-induced osteoclastogenesis was further confirmed.

Bone formation involves the proliferation, differentiation, and mineralization of osteoblasts. 7F2 osteoblastic cells normally begin to differentiate at day 4 , and the heightening of ALP activity is present as a marker of the earlier period of cell differentiation. Figure $6 \mathrm{~B}$ shows that ALP activities were clearly lower in 7F2 osteoblastic cells that had been treated with empty or curcuminoid-loaded liposomes. Furthermore, we monitored the mineralization of 7F2 osteoblastic cells by Alizarin Red S staining. Figure 6C and D shows that empty and curcuminoid-loaded liposomes inhibited the mineralization of 7F2 osteoblastic cells in a time-dependent fashion. In previous studies, Cur has been reported to inhibit the differentiation and mineralization of human or rat osteoblasts. $^{31,44}$ The concentration $(70 \mu \mathrm{M})$ of curcuminoid we loaded in liposomes was sevenfold higher than that of Cur $(5-10 \mu \mathrm{M})$ used in studies of the inhibition of osteoblast proliferation and differentiation. ${ }^{44,45}$ Although Cur-Lip suppressed osteoblast differentiation and mineralization, there were only minor effects on cell proliferation. This difference may be due to the varieties of cell types or the reduction of curcuminoid toxicity by liposome encapsulation. Further, we found that Cur-Lip exhibited relatively higher levels of ALP activity and mineralization in 7F2 osteoblastic cells than empty liposomes and BDMC-Lip. This finding has not been reported previously. It is unclear why liposomes suppressed osteoblast differentiation and mineralization. Zhang et al mentioned that lecithin (as phosphatidylcholine) can induce adipocyte differentiation in both 3T3-L1 and primary preadipocytes and that is consistent with our data presented in Figure S1. ${ }^{46}$ Furthermore, Diascro et al demonstrated that a high fatty acid content in rabbit serum induced adipocytelike differentiation in human and rat osteoblastic cells. ${ }^{47}$ Therefore, liposomes (lipid vesicles) can initiate the switch from osteoblasts to adipocyte-like cells. Interestingly, CurLip maintained osteoblast differentiation activity, whereas a reduction in activity was observed by the addition of empty liposomes and BDMC-Lip. Since Cur can inhibit adipocyte-like differentiation through the dephosphorylation of mitogen-activated protein kinase (MAPK) (ERK, JNK, and p38) phosphorylation, we propose that Cur-Lip can prevent lipid-induced inhibition of osteoblast differentiation but not BDMC-Lip. ${ }^{48}$

IL-1 $\beta$ is a very potent inflammatory inducer. Our study showed that empty and curcuminoid-loaded liposomes can successfully downregulate IL-1 $\beta$-induced COX-2 and MMP-3 expression in 7F2 osteoblasts and showed a high ratio of OPG/RANKL in the presence of IL-1 $\beta$ stimulation. However, empty liposomes and BDMC-Lip displayed a minor effect on the OPG/RANKL ratio in 7F2 osteoblasts in the absence of IL-1 $\beta$ stimulation. These results are in agreement with the findings of Mathy-Hartert et $\mathrm{al}^{49}$ who showed that Cur can inhibit IL- $1 \beta$-induced COX-2 and MMP-3 expression in human osteoblasts, and Guo et $\mathrm{al}^{50}$ who reported that BDMC can inhibit LPS-induced COX-2 expression. Furthermore, Treede et al demonstrated that phosphaticholines can inhibit TNF- $\alpha$-induced proinflammatory genes and NF- $\kappa \mathrm{B}$ activation in Caco- 2 cells. ${ }^{51}$ Therefore, empty liposomes can inhibit LPS-stimulated NO production and IL-1 $\beta$-induced COX-2 and MMP-3 expression because of the phosphaticholine content. To date, Cur-Lip can prevent bone loss more effectively than BDMC-Lip by increasing the ratio of OPG/RANKL and preventing lipid-induced inhibition of osteoblast differentiation. In the clinical study of Belcaro et al, Meriva, a curcuminphosphatidylcholine complex, was proven to decrease joint pain and improve joint function in 50 OA patients. ${ }^{52}$ Therefore, curcuminoid-loaded liposomes may have a promising effect against subchondral bone turnover and slow the progression of $\mathrm{OA}$.

\section{Acknowledgments}

This work was supported by research grants from the National Science Council (Taiwan) (NSC 102-2320-B-415-001, NSC 102-2320-B-367-001, and MOST 103-2320-B-367001-MY2) and Veterans Affairs Commission, Executive Yuan (Taiwan) (RVHCY101001, RVHCY102001, and RVHCY103005). 


\section{Disclosure}

The authors report no conflicts of interest in this work.

\section{References}

1. Kular J, Tickner J, Chim SM, Xu J. An overview of the regulation of bone remodelling at the cellular level. Clin Biochem. 2012;45:863-873.

2. Kearns AE, Kallmes DF. Osteoporosis primer for the vertebroplasty practitioner: expanding the focus beyond needles and cement. $A J N R$ Am J Neuroradiol. 2008;29:1816-1822.

3. Mulari MT, Qu Q, Härkönen PL, Väänänen HK. Osteoblast-like cells complete osteoclastic bone resorption and form new mineralized bone matrix in vitro. Calcif Tissue Int. 2004;75(3):253-261.

4. Roux S. New treatment targets in osteoporosis. Joint Bone Spine. 2010; 77:222-228.

5. Asagiri $\mathrm{M}$, Takayanagi $\mathrm{H}$. The molecular understanding of osteoclast differentiation. Bone. 2007;40:251-264.

6. Zupan J, Jeras M, Marc J. Osteoimmunology and the influence of pro-inflammatory cytokines on osteoclasts. Biochem Med (Zagreb). 2013;23:43-63.

7. Stein GS, Lian JB, van Wijnen AJ, et al. Runx 2 control of organization, assembly and activity of the regulatory machinery for skeletal gene expression. Oncogene. 2004;23:4315-4329.

8. Cooper LF. Biologic determinants of bone formation for osseointegration: clues for future clinical improvements. $J$ Prosthet Dent. 1998;80:439-449.

9. Thamamongood TA, Furuya R, Fukuba S, Nakamura M, Suzuki N, Hattori A. Expression of osteoblastic and osteoclastic genes during spontaneous regeneration and autotransplantation of goldfish scale: a new tool to study intramembranous bone regeneration. Bone. 2012;50:1240-1249.

10. Clérigues V, Guillén MI, Castejón MA, Gomar F, Mirabet V, Alcaraz MJ. Heme oxygenase-1 mediates protective effects on inflammatory, catabolic and senescence responses induced by interleukin- $1 \beta$ in osteoarthritic osteoblasts. Biochem Pharmacol. 2012;83:395-405.

11. Ginaldi L, Di Benedetto MC, De Martinis M. Osteoporosis, inflammation and ageing. Immun Ageing. 2005;2:14.

12. Leibbrandt A, Penninger JM. RANKL/RANK as key factors for osteoclast development and bone loss in arthropathies. Adv Exp Med Biol. 2009;649:100-113.

13. Romas E, Gillespie MT, Martin TJ. Involvement of receptor activator of NFkappaB ligand and tumor necrosis factor-alpha in bone destruction in rheumatoid arthritis. Bone. 2002;30:340-346.

14. Dougall WC. Molecular pathways: osteoclast-dependent and osteoclastindependent roles of the RANKL/RANK/OPG pathway in tumorigenesis and metastasis. Clin Cancer Res. 2012;18:326-335.

15. Cochran DL. Inflammation and bone loss in periodontal disease. J Periodontol. 2008;79:1569-1576.

16. Thomas GP, Baker SU, Eisman JA, Gardiner EM. Changing RANKL/ OPG mRNA expression in differentiating murine primary osteoblasts. J Endocrinol. 2001;170(2):451-460.

17. Yuan XL, Meng HY, Wang YC, et al. Bone-cartilage interface crosstalk in osteoarthritis: potential pathways and future therapeutic strategies. Osteoarthritis Cartilage. 2014;22(8):1077-1089.

18. Daheshia M, Yao JQ. The interleukin 1beta pathway in the pathogenesis of osteoarthritis. J Rheumatol. 2008;35:2306-2312.

19. Collin-Osdoby P. Regulation of vascular calcification by osteoclast regulatory factors RANKL and osteoprotegerin. Circ Res. 2004;95: 1046-1057.

20. Sharma AR, Jagga S, Lee SS, Nam JS. Interplay between cartilage and subchondral bone contributing to pathogenesis of osteoarthritis. Int J Mol Sci. 2013;14(10):19805-19830.

21. Jayaprakasha GK, Rao LJ, Sakariah KK. Antioxidant activities of curcumin, demethoxycurcumin and bisdemethoxycurcumin. Food Chem. 2006;98:720-724.

22. Tocharus J, Jamsuwan S, Tocharus C, Changtam C, Suksamrarn A. Curcuminoid analogs inhibit nitric oxide production from LPS-activated microglial cells. J Nat Med. 2012;66:400-405.
23. Rege S, Momin S, Wadekar S, Pratap A, Bhowmick D. Effect of demethoxycurcumin and bisdemethoxycurcumin on antioxidant activity of curcumin in refined sunflower oil. J Food Process Preserv. 2014;38(1):296-303.

24. Aggarwal BB, Harikumar KB. Potential therapeutic effects of curcumin, the anti-inflammatory agent, against neurodegenerative, cardiovascular, pulmonary, metabolic, autoimmune and neoplastic diseases. Int J Biochem Cell Biol. 2009;41:40-59.

25. Gupta SC, Patchva S, Koh W, Aggarwal BB. Discovery of curcumin, a component of golden spice, and its miraculous biological activities. Clin Exp Pharmacol Physiol. 2012;39(3):283-299.

26. Anand P, Kunnumakkara AB, Newman RA, Aggarwal BB. Bioavailability of curcumin: problems and promises. Mol Pharm. 2007;4:807-818.

27. Funk JL, Oyarzo JN, Frye JB, et al. Turmeric extracts containing curcuminoids prevent experimental rheumatoid arthritis. $J$ Nat Prod. 2006;69:351-355.

28. Bharti AC, Takada Y, Aggarwal BB. Curcumin (diferuloylmethane) inhibits receptor activator of NF-kappa B ligand-induced NF-kappa B activation in osteoclast precursors and suppresses osteoclastogenesis. J Immunol. 2004;172:5940-5947.

29. Hie M, Yamazaki M, Tsukamoto I. Curcumin suppresses increased bone resorption by inhibiting osteoclastogenesis in rats with streptozotocininduced diabetes. Eur J Pharmacol. 2009;621:1-9.

30. Kim JH, Gupta SC, Park B, Yadav VR, Aggarwal BB. Turmeric (Curcuma longa) inhibits inflammatory nuclear factor (NF)- $\mathrm{kB}$ and $\mathrm{NF}-\kappa \mathrm{B}-$-regulated gene products and induces death receptors leading to suppressed proliferation, induced chemosensitization, and suppressed osteoclastogenesis. Mol Nutr Food Res. 2012;56:454-465.

31. Notoya M, Nishimura H, Woo JT, Nagai K, Ishihara Y, Hagiwara H. Curcumin inhibits the proliferation and mineralization of cultured osteoblasts. Eur J Pharmacol. 2006;534:55-62.

32. Sandur SK, Pandey MK, Sung B, et al. Curcumin, demethoxycurcumin, bisdemethoxycurcumin, tetrahydrocurcumin and turmerones differentially regulate anti-inflammatory and anti-proliferative responses through a ROS-independent mechanism. Carcinogenesis. 2007;28:1765-1773.

33. Anand P, Nair HB, Sung B, et al. Design of curcumin-loaded PLGA nanoparticles formulation with enhanced cellular uptake, and increased bioactivity in vitro and superior bioavailability in vivo. Biochem Pharmacol. 2010;79:330-338.

34. Lin YL, Liu YK, Tsai NM, et al. A Lipo-PEG-PEI complex for encapsulating curcumin that enhances its antitumor effects on curcumin-sensitive and curcumin-resistance cells. Nanomedicine. 2012;8:318-327.

35. Suntres ZE. Liposomal antioxidants for protection against oxidantinduced damage. J Toxicol. 2011;2011:152474

36. Galović Rengel R, Barisić K, Pavelić Z, Zanić Grubisić T, Cepelak I, Filipović-Grcić J. High efficiency entrapment of superoxide dismutase into mucoadhesive chitosan-coated liposomes. Eur J Pharm Sci. 2002; 15:441-448.

37. Sun D, Zhuang X, Xiang X, et al. A novel nanoparticle drug delivery system: the anti-inflammatory activity of curcumin is enhanced when encapsulated in exosomes. Mol Ther. 2010;18:1606-1614.

38. Aditya NP, Chimote G, Gunalan K, Banerjee R, Patankar S, Madhusudhan B. Curcuminoids-loaded liposomes in combination with arteether protects against Plasmodium berghei infection in mice. Exp Parasitol. 2012;131:292-299.

39. Basnet P, Hussain H, Tho I, Skalko-Basnet N. Liposomal delivery system enhances anti-inflammatory properties of curcumin. J Pharm Sci. 2012;101:598-609.

40. Lietman SA, Yin L, Levine MA. SH3BP2 is an activator of NFAT activity and osteoclastogenesis. Biochem Biophys Res Commun. 2008; 371(4):644-648.

41. Furneri PM, Fresta M, Puglisi G, Tempera G. Ofloxacin-loaded liposomes: in vitro activity and drug accumulation in bacteria. Antimicrob Agents Chemother. 2000;44(9):2458-2464. 
42. Chen Y, Wu Q, Zhang Z, Yuan L, Liu X, Zhou L. Preparation of curcumin-loaded liposomes and evaluation of their skin permeation and pharmacodynamics. Molecules. 2012;17:5972-5987.

43. Jung KK, Lee HS, Cho JY, et al. Inhibitory effect of curcumin on nitric oxide production from lipopolysaccharide-activated primary microglia. Life Sci. 2006;79(21):2022-2031.

44. Moran JM, Roncero-Martin R, Rodriguez-Velasco FJ, et al. Effects of curcumin on the proliferation and mineralization of human osteoblast-like cells: implications of nitric oxide. Int J Mol Sci. 2012;13: 16104-16118.

45. Chan WH, Wu HY, Chang WH. Dosage effects of curcumin on cell death types in a human osteoblast cell line. Food Chem Toxicol. 2006; 44:1362-1371.

46. Zhang Y, Huang C, Sheng X, Gong Z, Zang YQ. Lecithin promotes adipocyte differentiation and hepatic lipid accumulation. Int J Mol Med. 2009;23:449-454.

47. Diascro DD Jr, Vogel RL, Johnson TE, et al. High fatty acid content in rabbit serum is responsible for the differentiation of osteoblasts into adipocyte-like cells. J Bone Miner Res. 1998;13(1):96-106.
48. Ahn J, Lee H, Kim S, Ha T. Curcumin-induced suppression of adipogenic differentiation is accompanied by activation of Wnt/beta-catenin signaling. Am J Physiol Cell Physiol. 2010;298(6):C1510-C1516.

49. Mathy-Hartert M, Jacquemond-Collet I, Priem F, Sanchez C, Lambert C, Henrotin Y. Curcumin inhibits pro-inflammatory mediators and metalloproteinase-3 production by chondrocytes. Inflamm Res. 2009;58:899-908.

50. Guo LY, Cai XF, Lee JJ, et al. Comparison of suppressive effects of demethoxycurcumin and bisdemethoxycurcumin on expressions of inflammatory mediators in vitro and in vivo. Arch Pharm Res. 2008;31(4): 490-496.

51. Treede I, Braun A, Sparla R, et al. Anti-inflammatory effects of phosphatidylcholine. J Biol Chem. 2007;282(37):27155-27164.

52. Belcaro G, Cesarone MR, Dugall M, et al. Efficacy and safety of Meriva ${ }^{\circledR}$, a curcumin-phosphatidylcholine complex, during extended administration in osteoarthritis patients. Altern Med Rev. 2010;15(4):337-344. 


\section{Supplementary material}

MM

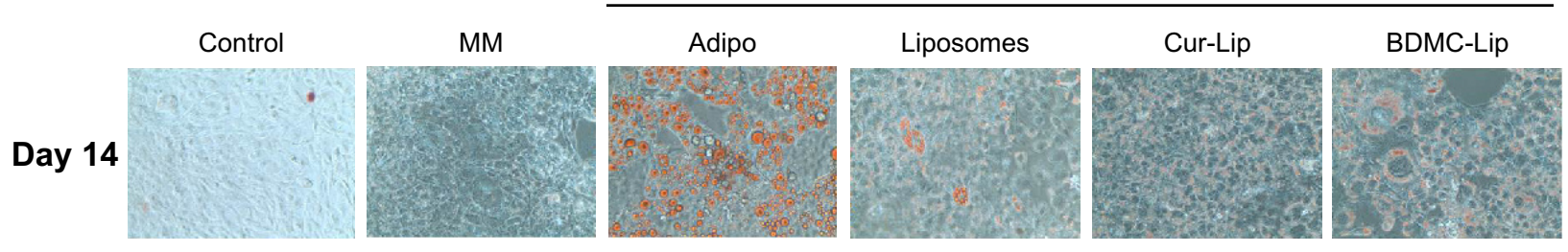

Figure SI Curcuminoid-loaded liposomes $(70 \mu \mathrm{M})$ induced adipogenesis in 7F2 osteoblastic cells after 7 days of cultivation.

Note: Adipo: cells were cultured in adipogenic differentiation medium for 7 days.

Abbreviations: BDMC-Lip, bisdemethoxycurcumin-loaded liposomes; Cur-Lip, curcumin-loaded liposomes; MM, mineralizing medium.

\section{Publish your work in this journal}

Drug Design, Development and Therapy is an international, peerreviewed open-access journal that spans the spectrum of drug design and development through to clinical applications. Clinical outcomes, patient safety, and programs for the development and effective, safe, and sustained use of medicines are a feature of the journal, which has also been accepted for indexing on PubMed Central. The manuscript management system is completely online and includes a very quick and fair peer-review system, which is all easy to use. Visit http://www.dovepress.com/testimonials.php to read real quotes from published authors.

Submit your manuscript here: http://www.dovepress.com/drug-design-development-and-therapy-journal 